\title{
A multicentre matched-pair analysis comparing robot-assisted versus open partial nephrectomy
}

\author{
Vincenzo Ficarra', Andrea Minervini ${ }^{2}$, Alessandro Antonelli ${ }^{3}$, Sam Bhayani ${ }^{4}$, \\ Giorgio Guazzoni ${ }^{5}$, Nicola Longo ${ }^{6}$, Giuseppe Martorana ${ }^{7}$, Giuseppe Morgia ${ }^{8}$, \\ Alexander Mottrie ${ }^{9}$, James Porter ${ }^{10}$, Claudio Simeone ${ }^{3}$, Gianni Vittori ${ }^{2}$, Filiberto Zattoni ${ }^{11}$ \\ and Marco Carini ${ }^{2}$
}

'Department of Urology, University of Udine, Udine, ${ }^{2}$ Department of Urology, University of Florence, Florence, ${ }^{3}$ Department of Urology, University of Brescia, Brescia, Italy, ${ }^{4}$ Washington University School of Medicine, Saint Louis, MO, USA, ${ }^{5}$ Department of Urology, Vita-Salute University, San Raffaele-Turro Hospital, Milan, ${ }^{6}$ Department of Urology, University Federico II, Naples, ${ }^{7}$ Department of Urology, University of Bologna, Bologna, Italy, ${ }^{8}$ Luna Foundation, ${ }^{9}$ OLV Robotic Surgery Institute, Aalst, Belgium, ${ }^{10}$ Swedish Urology Group, Seattle, WA, USA, and " Department of Urology, University of Padua, Padua, Italy

\section{Objective}

To compare the perioperative, pathological and functional outcomes in two contemporary, large series of patients in different institutions and who underwent open partial nephrectomy (OPN) or robot-assisted PN (RAPN) for suspected renal tumours.

\section{Patients and Methods}

This was a retrospective, multicentre, international, matched-pair analysis comparing patients who underwent RAPN or OPN for suspected renal cell carcinoma.

Data on patients who underwent OPN were extracted by an Italian observational registry collecting data from 19 different centres.

Data on patients who received RAPN were extracted from a multicentre, international database collecting cases treated in four high-volume referral centres of robotic surgery.

The matching was in a 1:1 ratio for the surgical approach and included 200 patients in each arm.

\section{Results}

The mean warm ischaemia time was shorter in the OPN group than in the RAPN group, at a mean (SD) of 15.4 (5.9) vs $19.2(7.3) \min (P<0.001)$.
Conversely, the median (interquartile range) estimated blood loss was $150(100-300) \mathrm{mL}$ in the OPN group and 100 $(50-150) \mathrm{mL}$ in the RAPN group $(P<0.001)$.

There were no differences in operating time $(P=0.18)$ and the intraoperative complication rate $(P=0.31)$ between the approaches.

Postoperative complications were recorded in 43 (21.5\%) patients who underwent OPN and in $28(14 \%)$ who received RAPN $(P=$ 0.02). Moreover, major complications (grade 3-4) were reported in nine (4.5\%) patients after OPN and in nine (4.5\%) after RAPN.

Positive margins were detected in nine (5.5\%) patients after OPN and in nine (5.7\%) after RAPN ( $P=0.98)$. The mean (SD) 3-month estimated glomerular filtration rate declined by 16.6 (18.1) $\mathrm{mL} / \mathrm{min}$ from the preoperative value in the OPN group and by $16.4(22.9) \mathrm{mL} / \mathrm{min}$ in the RAPN group $(P=0.28)$.

\section{Conclusion}

RAPN can achieve equivalent perioperative, early oncological and functional outcomes as OPN. Moreover, RAPN is a less invasive approach, offering a lower risk of bleeding and postoperative complications than OPN.

\section{Keywords}

kidney, partial nephrectomy, robotics, complications, renal tumour

\section{Introduction}

Partial nephrectomy (PN) is currently the 'gold-standard' treatment for localised renal tumours of $<7 \mathrm{~cm}$ [1]. PN is associated with lower renal function impairment [2], better overall survival and equivalent oncological survival compared with radical nephrectomy [3-5]. Therefore, the surgical feasibility according to the surgeon's expertise and preferences remains the main factor influencing the final decision.

In recent decades many authors have proposed laparoscopic PN (LPN) and robot-assisted PN (RAPN) as less invasive 
alternatives to open PN (OPN). Although LPN was able to offer better cosmetic results, less postoperative pain, shorter hospitalisation and postoperative recovery than OPN, the steep learning curve limited its diffusion to a few high-volume reference centres, and for small and less complex tumours [6].

RAPN has been developed and proposed as the natural evolution and simplification of LPN. Indeed, the advantages offered by the Da Vinci platform (Intuitive Surgical, CA, USA; three-dimensional vision and patented EndoWrist ${ }^{\mathrm{TM}}$ technology) can help surgeons to reduce the learning curve and to increase the feasibility of a laparoscopic approach also for the treatment of more complex and/or large renal tumours.

Recently, two meta-analyses of the results of the available studies comparing LPN and RAPN reported conflicting results. While Aboumarzouk et al. [7] reported a significant decrease of warm ischaemia time (WIT) in a RARP series, Froghi et al. [8] did not report significant differences in perioperative variables between the approaches. However, large comparative studies not included in the previous meta-analyses showed a significant advantage in favour of RAPN for WIT, estimated blood loss (EBL), hospital stay and risk of major complications $[9,10]$.

Some authors recently highlighted that LPN should not be considered as a suitable referent for RAPN [11]. Indeed, previous data convincingly support the concept that RAPN is a very attractive adaptation to duplicate the performance of OPN, even in more complex cases. However, only a few retrospective, single-centre studies have compared RAPN with OPN. These studies showed a longer WIT in the RAPN cohort but overlapping results for positive surgical margins (PSMs), perioperative complications and renal functional outcomes $[12,13]$.

In the present study, we used a matched-pair analysis between contemporary large series of patients in different institutions who underwent OPN or RAPN for suspected renal tumours. The aim of the study was to compare the perioperative, pathological and functional outcomes in the two cohorts.

\section{Patients and Methods}

Data of patients who underwent OPN (368) were extracted from the preliminary analysis of the REgistry of COnservative Renal surgery database (RECORd Project) promoted by the 'Leading Urological No profit foundation Advanced research' (LUNA) of the Italian Society of Urology (SIU) to collect the clinical records of patients who had PN for CT1 renal tumours between January 2009 and January 2011 at 19 urological centres in Italy. Data from patients who had RAPN were extracted from a multicentre, international database including 415 patients in four centres (Aalst, Belgium; Milan San Raffaele, Italy; Washington University School of Medicine, St
Louis, MO, USA; and Swedish Urology Group, Seattle, WA, USA), from September 2008 to September 2010.

Clinical staging included an abdominal CT and chest X-rays. MRI was used in a few patients as an alternative to CT. Bone scans and brain CT were obtained only when indicated by the signs and symptoms.

OPN procedures were performed by several expert surgeons using mainly a flank retroperitoneal approach. According to the surgeon's preference, the tumour was excised with (standard PN) or without (simple enucleation) an adequate rim of healthy renal parenchyma around the pseudocapsule. Tumour excision was done by clamping the renal artery (warm ischaemia) or with no clamping, using manual compression of the surrounding renal parenchyma. Opened calyces and bleeding sites were sutured and the parenchymal defect was closed with horizontal interrupted sutures with or without the application of haemostatic agents $\left(\mathrm{FloSeal}^{\circledR}\right.$, Baxter Healthcare; Tachosil ${ }^{\circledR}$, Nycomed; Tabotamp ${ }^{\circledR}$, Ethicon).

RAPN procedures were performed in high-volume centres by four surgeons (A.M., S.B., J.P., G.G.) with extensive robotic experience, including robotic radical prostatectomy, robotic radical nephrectomy and for some surgeons, robotic radical cystectomy. A transperitoneal approach was used in most cases. In all cases a standard PN was performed. The renal artery was clamped until the end of the renal parenchyma reconstruction in most cases. The renal vein was clamped only for more complex tumours. If the collecting system had been entered, or if large vessels remained patent, a repair with absorbable suture materials was made before proceeding with renorrhaphy. The parenchymal defect was repaired using the sliding-clip technique. If necessary, haemostatic agents were used.

The following preoperative variables were extracted from the databases: age, gender, clinical tumour size, polar location, tumour exophytic rate ( $>50 \%$ exophytic, $<50 \%$ exophytic, entirely endophytic), clinical TNM stage, preoperative blood haemoglobin and serum creatinine, and estimated GFR (eGFR) calculated with the Modification of Diet in Renal Disease equation [14].

The following intraoperative variables were evaluated: the use of pedicle clamping; WIT; operating time (calculated as the time from skin incision to skin closure); EBL; and intraoperative complications.

For every patient, the following pathological variables were recorded: tumour size, extension of the primary tumour according to the 2009 version of the TNM classification [15], histological subtypes according to the WHO classification [16], the nuclear grade according to the Fuhrman classification [17], and the surgical margin status. A PSM was defined as cancer cells at the level of the inked parenchymal excision surface. 
There was no central pathological slide review and the pathological features were assigned by the uro-pathologists of each participating centre.

The complications at 3 months after surgery were classified according to the Dindo modification of the Clavien system [18]. Postoperative complications were distinguished as minor (grade 1-2) and major (grade 3-4). The eGFR at 3 months after surgery was calculated to evaluate the potential effect of surgery on overall renal function.

The propensity score for matching was assessed using $\mathrm{R}$ Project, a method that controls for imbalances in confounding factors among discrete study cohorts $[19,20]$. The propensity score was calculated for each patient using multivariable logistic regression based upon the following covariates: age, clinical tumour size, longitudinal location (upper or inferior poles vs middle pole) and tumour exophytic rate $(<50 \%$ exophytic vs others). The matching was in a 1:1 ratio for the surgical approach (OPN vs RAPN) and included 200 patients in each arm.

Continuous parametric variables were reported as the mean \pm standard deviation (SD), while nonparametric variables were reported as the median and interquartile range (IQR). The Mann-Whitney $U$-test and unpaired Student's $t$-test were used to compare continuous variables, as appropriate. Categorical variables are reported as frequencies and proportions.

Pearson's chi-square test was used to compare categorical variables. For all statistical analyses, a two-sided $P<0.05$ was considered to indicate statistical significance.

\section{Results}

The preoperative characteristics of the patients are summarised in Table 1. For the laboratory variables, the haemoglobin $(P=0.94)$, serum creatinine $(P=0.99)$, and eGFR $(P=0.72)$ levels overlapped in the two groups of patients.

The renal artery was clamped in only 138 (69\%) patients who underwent OPN. Conversely, 180 (90\%) RAPNs were performed with warm ischaemia $(P<0.001)$. The median (IQR) WIT was 15 (11-19) and 18 (14-23) $\mathrm{min}$ for OPN and RAPN, respectively $(P<0.001)$. In detail, a WIT of $>20 \mathrm{~min}$ was recorded in $21(10.5 \%)$ patients after OPN and in 61 $(30.5 \%)$ after RAPN $(P<0.001)$. Only two $(1 \%)$ patients in the OPN group and $10(5 \%)$ in the RAPN group had a WIT of $>30$ min $(P=0.06)$. The median (IQR) EBL was $150(100-300)$ $\mathrm{mL}$ in the OPN group and $100(50-150) \mathrm{mL}$ in the RAPN group $(P<0.001)$. There were no differences in operating time $(P=0.19)$ and intraoperative complication rate $(P=0.31)$ between the approaches. Specifically, intraoperative complications included two venous injuries and one splenic injury in the OPN group, and one renal vein injury in the RAPN group. All previous complications were repaired during surgery without conversion to open surgery for the robotic procedures. Table 2 summarises the intraoperative data stratified according to the different surgical approaches. The baseline and postoperative mean (SD) haemoglobin levels (at 3 days after surgery) were $2.7(1.5) \mathrm{g} / \mathrm{dL}$ in the OPN group and $2(1.5) \mathrm{g} / \mathrm{dL}$ in the RAPN group $(P<0.001)$. Moreover, $20(10.3 \%)$ patients in the OPN group and $21(10.5 \%)$ in the RAPN group received a blood transfusion during the perioperative period $(P=0.78)$. The median (IQR) length of stay was 7 (6-8) days for OPN and 6 (5-6) days for RAPN $(P=0.014)$. Postoperative complications were recorded in 43 $(21.5 \%)$ patients who underwent OPN and in $28(14 \%)$ who had RAPN $(P=0.02)$. In detail, major complications (grade $3-4)$ were reported in nine (4.5\%) patients each after OPN and RAPN. Minor complications (grade 1-2) were recorded in

Table 1 Preoperative characteristics of patients and tumours stratified according to surgical approach.

\begin{tabular}{|c|c|c|c|}
\hline Preoperative variable & $\begin{array}{c}\text { RPN } \\
N=\mathbf{2 0 0}\end{array}$ & $\begin{array}{c}\text { OPN } \\
N=200\end{array}$ & $\boldsymbol{P}$ \\
\hline Mean (SD) age, years & $62.4(10.6)$ & $62.4(11.8)$ & 0.94 \\
\hline Gender, $n(\%)$ & & & 0.12 \\
\hline male & $121(60.5)$ & $131(65.5)$ & \\
\hline female & $79(39.5)$ & $69(34.5)$ & \\
\hline Median (IQR) clinical tumour diameter, $\mathrm{cm}$ & $2.8(1.9-3.5)$ & $3.0(2.0-3.5)$ & 0.13 \\
\hline Longitudinal tumour location, $n(\%)$ & & & 0.31 \\
\hline polar & $124(62)$ & $114(57)$ & \\
\hline non-polar & $76(38)$ & $86(43)$ & \\
\hline Percentage of tumour deepening into the kidney, $n(\%)$ & & & 0.45 \\
\hline$\geq 50 \%$ exophytic & $142(71)$ & $135(67.5)$ & \\
\hline$<50 \%$ exophytic/completely intrarenal & $58(29)$ & $65(32.5)$ & \\
\hline Clinical T stage, $n(\%)$ & & & 0.66 \\
\hline cTla & $171(85.5)$ & $174(87)$ & \\
\hline $\mathrm{cT} 1 \mathrm{~b}$ & $29(14.5)$ & $26(13)$ & \\
\hline \multicolumn{4}{|l|}{ Median (IQR): } \\
\hline Preoperative haemoglobin, g/dL & $14(13-15)$ & $14(13-15)$ & 0.94 \\
\hline Preoperative serum creatinine, $\mathrm{mg} / \mathrm{dL}$ & $1.0(0.8-1.1)$ & $0.9(0.8-1.1)$ & 0.99 \\
\hline Preoperative eGFR, $\mathrm{mL} / \mathrm{min}$ & $81.0(69.0-91.7)$ & $83.4(70.3-94.1)$ & 0.72 \\
\hline
\end{tabular}


Table 2 Intraoperative outcomes stratified according to the different surgical approach.

\begin{tabular}{lccc}
\hline Intraoperative variable & $\begin{array}{c}\text { RAPN } \\
\mathbf{N}=\mathbf{2 0 0}\end{array}$ & $\begin{array}{c}\text { OPN } \\
\mathbf{N}=\mathbf{2 0 0}\end{array}$ & $\boldsymbol{P}$ \\
\hline Artery clamping, $n$ (\%) & $180(90)$ & $138(69)$ & $<0.001$ \\
Median (IQR; range) WIT, min & $18(14-23 ; 5-51)$ & $15(11-19 ; 4-34)$ & $<0.001$ \\
$N(\%):$ & & & \\
WIT $>20$ min & $61(30.5)$ & $21(10.5)$ & $<0.001$ \\
WIT $>25$ min & $31(15.5)$ & $8(4)$ & 0.002 \\
WIT $>30$ min & $10(5)$ & $2(1)$ & 0.06 \\
Median (IQR) EBL, mL & $100(50-150)$ & $150(100-300)$ & $<0.001$ \\
Median (IQR) operating time, min & $120(90-157)$ & $3(1.5)$ & 0.19 \\
Intraoperative complications, $n(\%)$ & $1(0.5)$ & & 0.31 \\
\hline
\end{tabular}

Table 3 Perioperative outcomes stratified according to the different surgical approach.

\begin{tabular}{llcc}
\hline Variables & $\begin{array}{c}\text { RAPN } \\
\mathbf{N}=\mathbf{2 0 0}\end{array}$ & $\begin{array}{c}\text { OPN } \\
\mathbf{N}=\mathbf{2 0 0}\end{array}$ & $\boldsymbol{P}$ \\
\hline $\begin{array}{l}\text { Median (IQR) length of stay (including } \\
\text { day of surgery), days }\end{array}$ & $6(5-6)$ & $7(6-8)$ & 0.014 \\
$n / N(\%)$ : & & & \\
$\quad \begin{array}{l}\text { Postoperative overall complications } \\
\text { Clavien severity grade: }\end{array}$ & $28 / 200(14)$ & $43 / 200(21.5)$ & 0.027 \\
$1-2$ & $19(9.5)$ & $34(17)$ & 0.030 \\
3 & $8(4)$ & $7(3.5)$ & 0.34 \\
4 & $1(0.5)$ & $2(1)$ & - \\
Transfusion rate & $21 / 200(10.5)$ & $20 / 200(10)$ & 0.78 \\
\hline
\end{tabular}

$34(17 \%)$ patients after OPN and in 19 (9.5\%) after RAPN $(P=0.03)$. Table 3 details the perioperative outcomes stratified according to the two approaches.

The definitive histopathological examination showed 37 (17.5\%) benign tumours (angiomyolipomas or oncocytomas) in the OPN group and $42(21 \%)$ in the RAPN group, respectively $(P=0.63)$. there were no significant differences in pathological extension of the primary tumour $(P=0.54)$ and Fuhrman nuclear grade $(P=0.72)$. PSMs were detected in nine $(5.5 \%)$ patients after OPN and in nine $(5.7 \%)$ after RAPN $(P=0.98)$. Table 4 summarises the pathological features according to the two approaches.

At 3 months after surgery, the mean (SD) eGFR declined by $16.6(18.1) \mathrm{mL} / \mathrm{min}$ from the preoperative value in the OPN group and by $16.4(22.9) \mathrm{mL} / \mathrm{min}$ in the RAPN group $(P=0.28)$.

\section{Discussion}

The present matched-pair analysis showed that RAPN is a valid alternative to OPN in patients with suspected cT1 renal tumours. Although OPN offered the advantages of a higher percentage of operations without renal artery clamping and a shorter WIT than RAPN, the robot-assisted approach was associated with a statistically significantly lower risk of blood loss (although this was probably not clinically relevant), of postoperative complications, and with a shorter hospital stay.
Table 4 Pathological features stratified according to the different surgical approach.

\begin{tabular}{|cccc}
\hline Variables & RAPN & OPN & P \\
& $\mathbf{N}=\mathbf{2 0 0}$ & $\mathbf{N = \mathbf { 2 0 0 }}$ & \\
\hline$n / N(\%):$ & & $37(17.5)$ & 0.63 \\
Benign tumours & $42(21)$ & & 0.54 \\
TNM 2009: & & $126 / 163(77.3)$ & \\
pT1a & $132 / 158(83.5)$ & $24 / 163(14.7)$ & \\
pT1b & $18 / 158(11.4)$ & - & \\
pT2 & - & $13 / 163(8.0)$ & \\
pT3a & $8 / 158(5.1)$ & & \\
Fuhrman grade: & & $33 / 163(20.2)$ & \\
1 & $25 / 158(15.8)$ & $97 / 163(59.5)$ & \\
2 & $97 / 158(61.4)$ & $33 / 163(20.2)$ & \\
3-4 & $36 / 158(22.8)$ & $9 / 163(5.5)$ & 0.98 \\
PSM & $9 / 158(5.7)$ & & \\
\hline
\end{tabular}

The rate of PSMs, and early functional outcomes evaluated using the eGFR, at 3 months after surgery showed overlapping results between the techniques.

Most of the comparative studies reported previously tested RAPN vs LPN. Two recent meta-analyses showed only minimal advantages in favour of RAPN in terms of a shorter WIT $[7,8]$. However, both meta-analyses provided a combination of data coming from small, observational studies in which the LPN group was represented by historical series and the RAPN group was strongly influenced by the surgeons' learning curve [21]. Moreover, the two larger comparative studies were not included in both meta-analyses for methodological reasons. Both studies showed significant advantages in favour of RAPN for WIT, EBL, hospital stay $[9,10]$ and the risk of major complications [10].

Similarly, only a few observational studies comparing RAPN and OPN have been reported. In 2011, Lee et al. [12] retrospectively compared 69 RAPN and 234 OPN procedures in patients comparable in age, gender, clinical tumour size and preoperative eGFR. Although the mean WIT was longer in the RAPN group, no significant differences in postoperative eGFR and change in the eGFR were detected. Moreover, the hospital stay, use of analgesics and overall postoperative complication rates were significantly in favour of the RAPN group [12]. The 
previous favourable outcomes were confirmed also by Simhan et al. [13] in 2012, comparing the outcomes of 91 RAPN and 190 OPN for moderately and highly complex renal lesions, according to R.E.N.A.L. nephrometry [consists of (R)adius (tumour size as maximal diameter), (E)xophytic/endophytic properties of the tumour, $(\mathrm{N})$ earness of tumour deepest portion to the collecting system or sinus, (A)nterior (a)/posterior (p) descriptor and the (L)ocation relative to the polar line]. Indeed, in this non-matched-pair analysis, the authors showed that RAPN offered comparable perioperative and functional outcomes with a shorter hospital stay than OPN [13]. In the same year, Lucas et al. [22], in a small, retrospective, comparative study analysing 27 RAPN and 54 OPN, showed a shorter WIT in the OPN group but a greater blood loss and hospital stay than in the RAPN group. Perioperative complications and renal functional preservation were similar for each treatment.

Similar to the present study, in a small matched-pair analysis including only 27 RAPN and 54 OPN, Mellon et al. [23] reported overlapping PSM rates between RAPN and OPN. Moreover, the authors showed that the mean distance to the proximal margin edge for RAPN specimens was equivalent to those in OPN [23].

Considering the clinical characteristics of patients included in the present study, our conclusions should be further confirmed in other studies enrolling a higher percentage of cT1b tumours. In 2013, Sprenkle et al. [24] compared minimally invasive (LPN and RAPN) PN and OPN for the treatment of cT1b renal tumours. Although that study concluded that minimally invasive PN procedures offer acceptable and comparable results for operative, functional and convalescence measures, the conclusions were based on only 16 robotic procedures evaluated retrospectively.

The present study has some limitations. Although the matched-pair analysis allowed control for imbalanced factors among the two study cohorts, this was not a randomised controlled trial. Moreover, the two cohorts were not adjusted for some potential confounding factors, e.g. patient comorbidity. However, the two cohorts were matched for age and were not significantly different in preoperative haemoglobin and serum creatinine levels. The two groups were also not adjusted for tumour characteristics included in the most used nephrometry systems, e.g. renal rim location (medial or lateral), renal sinus and collecting system involvement, and face location (anterior or posterior). Another limitation is the lack of some anatomical information in the RECORD database generated in 2008, before the publication of the most common nephrometry systems (R.E.N.A.L. nephrometry and Preoperative Aspects and Dimensions Used for an Anatomical [PADUA] classification) [25,26]. Considering the observational design of both the RECORD project and PN robotic database, as well as the number of centres and surgeons involved, the surgical approaches and techniques resulted in heterogeneity. However, this potential limitation reflects the real-life situation in which patients are usually treated. For these reasons the RECORd 2 project was started in January 2013, including both PADUA and R.E.N.A.L. nephrometry scores, as well as the American Society of Anesthesiologists physical status classification system, Charlson score and age-adjusted Charlson score.

A further limitation is represented by the potential effect of the learning curve on the outcomes. Whilst most patients underwent OPN conducted by expert surgeons, some data extracted from the RAPN databases were the initial cases operated by the four participating surgeons. Therefore, according to the Idea, Development, Exploration, Assessment, Long-term follow-up (IDEAL) criteria, this study cannot be considered formally as an 'Assessment study' comparing RAPN with the surgical 'gold standard' (OPN), but it should be considered in the context of the evaluation step of the RAPN [27].

In conclusion, the present study showed that RAPN can attain equivalent perioperative and functional outcomes as OPN in patients with suspected cT1 renal tumours. Moreover, RAPN is a less invasive approach, offering a lower risk of bleeding and postoperative complications than OPN. However, the latter procedure is associated with a shorter WIT and a higher percentage of unclamped procedures. The overall renal function evaluated at 3 months after surgery seems to be equivalent between the approaches. The results in the RAPN group could be negatively influenced by the learning curve of the surgeons. Indeed, some initial robotic cases could possibly have been included in the matching analysis of the 200 cases and then compared with the 200 OPN cases. Therefore, further studies excluding patients operated during the learning curve should be planned to assess definitively the performance of RAPN vs OPN. Moreover, the present results should not be applied to small-volume robotic centres and to inexperienced users of the Da Vinci system.

\section{Conflict of Interest}

None declared.

\section{References}

1 Ljungberg B, Cowan NC, Hanbury DC et al. EAU guidelines on renal cell carcinoma: the 2010 update. Eur Urol 2010; 58: 398-404

2 Huang WC, Levey AS, Serio AM et al. Chronic kidney disease after nephrectomy in patients with renal cortical tumours: a retrospective cohort study. Lancet Oncol 2006; 7: 735-40

3 MacLennan S, Imamura M, Lapitan MC et al. Systematic review of oncological outcomes following surgical management of localized renal cancer. Eur Urol 2012; 61: 972-93

4 Van Poppel H, Da Pozzo L, Albrecht W et al. A prospective, randomised EORTC intergroup phase 3 study comparing the oncologic outcome of elective nephron-sparing surgery and radical nephrectomy for low-stage renal cell carcinoma. Eur Urol 2011; 59: 543-52 
5 Antonelli A, Ficarra V, Bertini R et al. Elective partial nephrectomy is equivalent to radical nephrectomy in patients with clinical T1 renal cell carcinoma: results of a retrospective, comparative, multi-institutional study. BJU Int 2012; 109: 1013-8

6 Ficarra V, Bhayani S, Porter J et al. Predictors of warm ischemia time and perioperative complications in a multicenter, international series of robot-assisted partial nephrectomy. Eur Urol 2012; 61: 395-402

7 Aboumarzouk OM, Stein RJ, Eyraud R et al. Robotic versus Laparoscopic Partial Nephrectomy. A systematic review and meta-analysis. Eur Urol 2012; 62: 1023-33

8 Froghi S, Ahmed K, Khan MS, Dasgupta P, Challacombe B. Evaluation of robotic and laparoscopic partial nephrectomy for small renal tumours (T1a). BJU Int 2013; 112: E322-33

9 Benway BM, Bhayani SB, Rogers CG et al. Robot assisted partial nephrectomy versus laparoscopic partial nephrectomy for renal tumors: a multi-institutional analysis of perioperative outcomes. J Urol 2009; 182: 866-73

10 Mullins JK, Feng T, Pierorazio PM, Patel HD, Hyams ES, Allaf ME. Comparative analysis of minimally invasive partial nephrectomy techniques in the treatment of localized renal tumors. Urology 2012; 80: $316-21$

11 Mottrie A, Borghesi M, Ficarra V. Is traditional laparoscopy the real competitor of robot-assisted partial nephrectomy? Eur Urol 2012; 62: $1034-6$

12 Lee S, Oh J, Hong SK, Lee SE, Byun SS. Open versus robot-assisted partial nephrectomy: effect on clinical outcome. J Endourol 2011; 25: 1181-5

13 Simhan J, Smaldone MC, Tsai KJ et al. Perioperative outcomes of robotic and open partial nephrectomy for moderately and highly complex renal lesions. J Urol 2012; 187: 2000-4

14 Huang WC, Elkin EB, Levey AS, Jang TL, Russo P. Partial nephrectomy versus radical nephrectomy in patients with small renal tumors-is there a difference in mortality and cardiovascular outcomes? J Urol 2009; 181: $55-61$

15 Greene FL, Gospodarowicz M, Wittekend C et al. American Joint Committee on Cancer (AJCC) Staging Manual, 7th edn. Philadelphia, PA: Springer, 2009

16 Eble JN, Sauter G, Epstein JI et al eds. Pathology and Genetics of Tumors of the Urinary System and Male Genital Organs. World Health Organization Classification of Tumors. Lyon, France: IARC Press, 2004

17 Fuhrman S, Lasky LC, Limas L. Prognostic significance of morphologic parameters in renal cell carcinoma. Am J Surg Pathol 1982; 6: 655-63
18 Dindo D, Demartines N, Clavien PA. Classification of surgical complications: a new proposal with evaluation in a cohort of 6,336 patients and results of a survey. Ann Surg 2002; 240: 205-13

19 Rosenbaum PR, Rubin DB. The central role of the propensity score in observational studies for causal effects. Biometrika 1983; 70: 41-55

20 Sekhon JS. Multivariate and Propensity Score Matching Software with Automated Balance Optimization: The Matching Package for R. J Stat Soft 42: $7(2011 / 5)$

21 Ficarra V, Novara G, Volpe A, Mottrie A. Robot-assisted Versus Traditional Laparoscopic Partial Nephrectomy: the time for meta-analysis is not yet coming. BJU Int 2013; 112: E334-6

22 Lucas SM, Mellon MJ, Erntsberger L, Sundaram CP. A comparison of robotic, laparoscopic and open partial nephrectomy. JSLS 2012; 16: $581-7$

23 Mellon MJ, Lucas SM, Kum JB, Cheng L, Sundaram C. A comparison of pathologic outcomes of matched robotic and open partial nephrectomies. Int Urol Nephrol 2013; 45: 381-5

24 Sprenkle PC, Power N, Ghoneim T et al. Comparison of open and minimally invasive partial nephrectomy for renal tumors 4-7 centimeters. Eur Urol 2012; 61: 593-9

25 Kutikov A, Uzzo RG. The R.E.N.A.L. nephrometry score: a comprehensive standardized system for quantitating renal tumor size, location and depth. J Urol 2009; 182: 844-53

26 Ficarra V, Novara G, Secco S et al. Preoperative aspects and dimensions used for an anatomical (PADUA) classification of renal tumors in patients who are candidates for nephron-sparing surgery. Eur Urol 2009; 56: 786-93

27 McCulloch P, Altman DG, Campbell WB et al. No surgical innovation without evaluation: the IDEAL recommendations. Lancet 2009; 374: $1105-12$

Correspondence: Andrea Minervini, Department of Urology, Careggi Hospital, Villa Monna Tessa, Viale Pieraccini 18, 50139, Florence, Italy.

e-mail: andreamine@libero.it

Abbreviations: EBL, estimated blood loss; eGFR, estimated GFR; IQR, interquartile range; $(\mathrm{O})(\mathrm{L})(\mathrm{RA}) \mathrm{PN}$, (open) (laparoscopic) (robot-assisted) partial nephrectomy; PSM, positive surgical margins; WIT, warm ischaemia time. 